\title{
Understanding the Facts and Minding the Gap of (HIV-1/HIV-2) Primate Research and Infectious Disease Laboratories in Africa
}

\author{
Chika Ejikeugwu ${ }^{1,2,3^{*}}$, Peter Eze ${ }^{4}$, Ifeanyichukwu Iroha ${ }^{1}$, Charles Esimone ${ }^{4}$, Michael Adikwu $^{5}$ and \\ Hirofumi Akari ${ }^{2,3}$
}

${ }^{1}$ Department of Applied Microbiology, Faculty of Science, Ebonyi State University, Nigeria

${ }^{2}$ Center for Human Evolution Modeling Research, Primate Research Institute, Kyoto University, Japan

${ }^{3}$ Laboratory of Infectious Disease Model, Institute for Frontier Life and Medical Sciences, Kyoto University, Japan

${ }^{4}$ Department of Pharmaceutical Microbiology and Biotechnology, Faculty of Pharmaceutical Sciences, Nnamdi Azikiwe University, Nigeria

${ }^{5}$ Department of Pharmaceutics, Faculty of Pharmacy, University of Nigeria Nsukka (UNN), Nigeria

*Corresponding author: Chika Ejikeugwu, Ph.D, Department of Applied Microbiology, Faculty of Science, Ebonyi State University, Abakaliki, P.M.B 053, Ebonyi State, Nigeria; Center for Human Evolution Modeling Research, Primate Research Institute; Laboratory of Infectious Disease Model, Institute for Frontier Life and Medical Sciences, Kyoto University, Kyoto, Japan, Tel: +2347081775676

\begin{abstract}
With over 2 million annual new infections of human immunodeficiency virus (HIV)-1, and more than 30 million HIV-1 infected people around the globe, the HIV-1 pandemic has continued to remain a front burner in the medical research community and an important public health debate. Till date, there is still no cure or vaccine for HIV-1 infection. Though current antiretroviral therapy (ART) is potent enough to reduce viral load of the infection to below detectable limits (< 50 copies $/ \mathrm{ml}$ ), antiretroviral therapy (ART) still lacks the capacity to cure or eradicate HIV-1. This inability of ART to either cure or eradicate HIV-1 is largely attributable to the non-functionality of ART to reach some perceived cellular and anatomical reservoir sites - where latent HIV-1 is lurking and getting protection from both host immunological response and the pharmacological attack of potent ART. Reservoir sites for latent HIV-1 are established soon after infection, and they remain hidden from immune responses of host and ART attack. These reservoir sites continue to serve as repertoire for continuous HIV-1 replication and viral production that initiates new infection even in the face of potent ART and in most part, off-ART. This is the bane and major obstacle to HIV-1 cure and eradication. However, with continuous research targeted at characterizing the various HIV-1 reservoir sites, it is believed and expected that novel ART will be discovered to target and dislodge the latent virus from their hiding places. While HIV-1 related research is
\end{abstract}

intensified in Europe, Americas and Asia; Africa is still lagging behind as a major contributor to finding a cure or vaccine for the disease. This lacuna in HIV-1-related research in Africa is largely attributable to paucity of funding and collaboration, lack of research facilities and the lack of political willpower to sponsor researchers to investigate this public health dilemma (i.e., HIV-1 infection) that the continent shares a huge part of the disease burden. This review sheds light on a general understanding of HIV-1 reservoir, some current cure strategies, latency reversing agents (LRAs) and the need for Africa to bridge the gap of primate and HIV-1 related research in the region, since Africa is a key beneficiary of the world's HIV-1 epidemic burden.

\section{Keywords}

HIV, AIDS, SIV, Reservoir sites, Cure research, Non Human Primate, Africa

\section{Introduction}

In this review, we reviewed the concept of HIV-1 reservoir and cure, non human primate (NHP) models for HIV-1-related research, LRAs and the need for Africa to resuscitate and sustain HIV-1-related research, especially those targeted at finding a cure for HIV-1,

Citation: Ejikeugwu C, Eze P, Iroha I, Esimone C, Adikwu M, et al. (2018) Understanding the Facts and Minding the Gap of (HIV-1/HIV-2) Primate Research and Infectious Disease Laboratories in Africa. Int J Virol AIDS 5:046. doi.org/10.23937/2469-567X/1510046

Accepted: December 29, 2018: Published: December 31, 2018

Copyright: (c) 2018 Ejikeugwu C, et al. This is an open-access article distributed under the terms of the Creative Commons Attribution License, which permits unrestricted use, distribution, and reproduction in any medium, provided the original author and source are credited. 
the causative agent of acquired immunodeficiency syndrome (AIDS). Since HIV-1 has a very short half-life in the blood plasma, why is HIV infection still not curable by HAART (highly active antiretroviral therapy) or the currently used combination antiretroviral therapy (cART)? It is because of this question that we are meant to believe in part, that latent HIV-1 still persists in some cells, tissues and organs of the body even in the face of potent ART; while waiting for opportunity to breakout from their hiding and initiate new infections in already infected individuals. These sanctuaries for latent HIV-1 (known in the most part of this review as reservoir sites) act as sources of new virus that initiate new infection when therapy is interrupted by infected individuals.

Unlike HIV-1 which is a worldwide epidemic, HIV-2 is not spread worldwide. HIV-2 is the less-widespread relative of HIV-1; and its pathological conditions are usually restricted to the sub-Saharan continent and some other parts of the world where it may have spread to $[1,2]$. The persistence of HIV-1 in infected individuals despite HAART is attributed to reservoirs of latently infected CD4+ memory $T$ cells and other reservoir anatomical sites of HIV-1 such as lymph nodes that are not easily permissible to ART and the infected hosts' immunological mechanisms [1-6]. AIDS is a retroviral disease that has global reach but Africa faces the disease most acutely. The reason being that, Africa accounts for the largest number of people infected by HIV-1 around the globe, aside the population of people who are also infected with HIV-2; and the region is battling with its health system which is still underdeveloped in most parts. More so, the level of HIV-1 related research in Africa is at a pitiable state due to lack of researchers, research facilities and proper funding to investigate this unique disease burden.

It is therefore critical that the continent sees it as a responsibility to be on the frontline of garnering research activities that will lead to the possible cure and eradication of HIV-1 and its less-pathogenic counterpart (HIV-2) as well as the total cure and eradication of other infectious diseases such as Lassa fever, Ebola and malaria to mention a few, that ravages the people of the region. Finding a cure for HIV-1 infection and/or AIDS is still an outstanding medical quagmire that has resisted all known current and sophisticated medical interventions, research and therapeutic applications. Africa is leading in the number of people infected with the less-pathogenic strain of HIV (i.e., HIV-2) - which is mainly endemic in West Africa; and the region is still lacking behind in terms of the number of primate research institutes (PRIs) and other high-tech infectious diseases laboratories or facilities for studying and proffering solutions to the continents infectious diseases.

In this review, we succinctly explained the general principles of latent HIV-1 reservoir sites as a way of awakening the interest of HIV-1-related researchers in this part of the world. The concepts of latent HIV-1, viral rebound, LRAs, HIV-1 and HIV-2, and the global burden of HIV/AIDS distribution - with a focus on the African continent are also highlighted. Besides, we also discussed briefly the role and importance of gene therapy techniques in the possible cure and eradication of HIV-1. This review also shed some light on the biological (animal) models used in HIV/AIDS related research and the need for Africa to be a major player in HIV/AIDS-related research - since it is also a major beneficiary of the global HIV-1 disease burden.

\section{HIV-1 Research is Targeted at Cure and Eradi- cation: Latency Reversing Agents}

In countries with the highest occurrence of HIV-1 infections (particularly in Sub-Saharan Africa), the life expectancy and total well-being of people living with HIV/ AIDS (PLHA) is being impoverished due to the pitiable state of health infrastructure and poor access to quality healthcare especially for HIV-1 infected individuals. The poor standard of living in the region coupled with the comatose health care sector puts the life of PLHA at much risk - compared to their counterparts in other developed economies with resources to research into finding a functional cure for HIV/AIDS. With the African continent taking the lead in the current prevalence of HIV/ AIDS in the world, and with the paucity of high-throughput research facilities in the region, it is important that leaders and researchers and health care policy makers in the region sees the need to set up more infectious disease laboratories and PRIs that will act as centers of excellence for infectious disease research. Such centers will not only research into HIV-1 reservoir and cure, but that will also help to carry out timely and targeted research whose output will help to proffer sustainable cure, vaccines, prevention and treatment strategies to Africa's own diseases such as malaria, Lassa and Ebola to mention a few. Currently, there is serious paucity of high-throughput infectious disease laboratory in Africa that will independently investigate into finding a cure or vaccine for HIV/AIDS. Only few researchers and institutions in Africa are looking towards HIV-1 research; and these individuals and/or institutions usually have collaborations outside the continent, where research facilities and funds are always available for such high-throughput medical research.

The search for a cure and vaccine for HIV-1 infection is still ongoing and putative. The widely reported cure of HIV-1 in Europe (the Berlin patient scenario) using haematopoietic stem cell transplantation technique, and in addition to many other outstanding findings targeted at acknowledged futuristic HIV/AIDS cure raises the hope that a sustainable/functional cure for HIV-1 infection is nearer than we thought despite the fact that latent HIV-1 still persist in the infected host as quiescent (latent) virus [7-9]. Understanding the molecular mechanisms underlying this possible cure 
strategy as well as the immunological and anatomical dispositions of HIV-1 reservoir cells and tissues in the already infected hosts will help us to achieve a possible remission of the virus from the body of PLHA. Even if not a total remission, HIV/AIDS cure strategies can be reengineered to teach the infected host's immune system on how to target more effectively and possibly flush out latent HIV-1 from latently infected host cells especially after the usage or administration of latency reversing agents (LRAs) and other potent antiretrovirals that has the capabilities to re-activate quiescent forms of the virus.

LRAs are now the in-thing in HIV-1 related research targeted at a functional cure of the infection. LRAs are defined as drugs or chemical compounds that reactivate viral production [10] in vivo or in vitro; and especially from their hiding reservoir sites in the body of PLHA. They are small pharmacological molecules that could help uncover where the provirus or latent HIV-1 is hiding in the cells or tissues of HIV-1 infected individuals whose viral load has been suppressed below the level of treatment by effective HAART/ART. The advent of HAART cum cART has significantly improved the life expectancy of PLHA due to its ability to suppress viral replication for long periods of time; but HAART/ART does not cure HIV1 , and it has some untoward effects that may affect the quality of life of HIV-1 infected individuals. Thus, LRAs are being intensively pursued experimentally, in order to come up with novel therapeutic strategies of purging reservoir sites of the latent virus, and possibly leading to viral remission and eradication in PLHA. It is however unfortunate that research targeted at finding novel LRAs for HIV-1 infection is still at its lowest ebb if not missing in Africa, a continent with the world's highest number of PLHA.

Why LRAs? ART has only succeeded in making HIV-1 infection a treatable disease but not yet curable; and this is due in part to the fact that ART has the ability to reduce plasma viral load of HIV-1 to below detectable limits (usually < 40-50 copies $/ \mathrm{ml}$ ) in peripheral circulation. Since ART can only reduce plasma viral load of the infection but not cure or eradicate the infectious virus from the body of PLHA, there arose the need for agents capable of dislodging the infectious virus from their reservoir sites in the body of PLHA and who are on ART. LRAs are one of such potent agents currently being investigated as possible flushing (curing) agents for possible HIV-1 cure.

The major goal of proposing for the use of LRAs in HIV-1 infection is to reactivate the latent reservoir of the virus in vivo so that infected cells or tissues (acting as sanctuaries for latent HIV-1) can be recognized and targeted by the host immune system, with the ultimate goal of achieving an HIV-1 cure [11,12]. LRAs include histone deacetylase inhibitors (HDACis); some growth factors (IL-15); checkpoint blockades (programmed cell death -1, PD-1); protein kinase C (PKC), and disulfiram (used to treat chronic alcoholism) to mention a few [6]. HDACis include vorinostat, panobinostat, romidepsin and entinostat. And which is mainly responsible for the intermittent chronic infection seen in PLHA even in the face of ART or off-ART. The use of small RNAs that use RNA interference pathway to target HIV-1 replication in vivo - as a possible functional cure approach for HIV1 remission has also been proposed [13]. Aside this, the targeting of the Vif protein in HIV-1 to activate the APOBEC antiviral pathway is another HIV-1 cure strategy known as the silencing strategy that is currently being proposed as a cure strategy [14].

In the study by Darcis, et al. [15], in which LRAs was investigated both in vitro and in vivo to compare the efficacy of various HIV-1 latency models, it was reported that bryostatin-1 + JQ1 and ingenol-B + JQ1 (which are potential LRAs still being investigated as possible HIV-1 cure or reservoir sites purging-agents) had more possibility or potential of reactivating viral gene expression. As aforementioned, HIV reservoir and cure research is now the 'in thing' in HIV/AIDS related research since it is mainly geared towards understanding the sanctuaries of latent HIV-1 in the cells and tissues of infected human and/or animal host, thereby coming up with specific clinical strategies to purge these safe haven for quiescent (latent) HIV-1. The lodgment or hiding of quiescent HIV-1 strains in specific host cells, tissues and organs where current medications and the host immune system armamentarium cannot reach them is a major impediment to the cure of HIV $[6,10]$.

Ever since its discovery in the early 1980s, mankind is still battling with this pandemic (HIV/AIDS) that has defiled almost every medical intervention developed for its possible cure and total elimination from the global community. LRAs are very non-specific in their ability to activate genes, causing a lot of off-target consequences. But LRAs may hold some potential to cure or eradicate the virus if properly harnessed to be selectively toxic in its in vivo activities in PLHA. Diminishing the size and amount of HIV reservoirs in major HIV-1 sanctuaries in the body is indispensable to finding a lasting cure for HIV. And this could be achieved if current cure strategies (especially the maximization of the potentials of LRAs and other putative cure strategies) and animal models used for studying the progression of the diseases across different centers in the world are properly coordinated and financially supported as much as possible. Research targeted at HIV-1 cure strategies is lacking in Africa due in part to lack of funding and unavailability of research facilities and technical know-how to undertake such studies. Since the top most priority of the International AIDS Society (IAS) is "Towards an HIV Cure"; it is important to find out and develop novel (less-toxic) and affordable-sustainable measures that will help to purge HIV-1 reservoir sites of the infectious (quiescent) virus through effective and sustainable research geared 
towards finding a total cure for the disease. And Africa being a major beneficiary of the global HIV/AIDS burden must not be left in the dark in this important medical pursuit. She must take the bull by the horn and strategize on how to institutionalize modern and high-tech or high-throughput research facilities across the continent in order to not only research into HIV/ AIDS but also lead ground breaking researches into the continent's own diseases such as Lassa fever, Ebola and malaria to mention a few. This will go a long way to bridging the research gap that exists in the continent as far as HIV/AIDS research and other infectious diseases as aforesaid are concerned. But while cure and eradication strategies are pursued with HIV-1 strains in mind, it is also important to take into cognizance the counterpart of this widely distributed virus, (i.e., HIV-2) - which is peculiar to West Africa - since the relatedness of HIV-1 and HIV-2 might be sufficient enough to spark some genetic recombination that might produce a different virulent and infectious HIV strain in the future. In this case, it is better to be prepared for such phenomenon by pursuing HIV-2 research in line with its globally distributed strain, HIV-1. The following section summarizes HIV-1 reservoir sites and viral rebound.

\section{Latent HIV-1 Reservoir Sites and Viral Rebound}

Latent HIV reservoirs are certain cells, tissues or organs of the body that harbour replication competent but transcriptionally silent HIV-1 strain(s) that are not actively producing new virions (especially in the presence of ART) or viral proteins (particles) that could be easily targeted by ART or the hosts' immunological machinery for possible purging and destruction. Latent HIV-1 have also been previously described as a replication competent but transcriptionally-silent HIV-1 that persists in HIV-1 infected individuals for a long time and during the period of active ART, but also has the ability to resume replication upon the discontinuance of ART $[5,7]$. According to Cary and Peterlin [6], latent
HIV-1 does not replicate or produce viral proteins, and this prevents the efficient targeting of the virus in its sanctuaries or reservoir sites by potent ART as well as by the hosts' immune system. This perhaps is one of the most challenging factors in the fight against HIV/ AIDS since latent HIV-1 strains are secluded and given protection by these reservoir cells so that ART or the host immune system will not reach them.

A viral reservoir site is a cell type or anatomical site in the body of PLHA, where a replication-competent form of latent HIV-1 can accumulate and continue stably [16]. HIV-1 reservoir sites represent one of the major impediment to the cure or eradication of HIV-1 from the body of PLHA - since these cellular/anatomical sites continue to serve as sources from which infectious virus are released into the peripheral circulation to initiate new infection even in individuals on ART. When ART is interrupted in HIV-1 infected individuals, viral rebound of new HIV-1 infection is bound to occur; and in developing countries where treatment and health care is still pitiable, the situation could worsen - with already infected people having rapid viraemia coupled with other co-morbidities associated with HIV-1 infection. The poor state of health facilities and infrastructure in Africa may impede on the proper management of PLHA in the region, and thus worsen the prognosis of such individuals especially in the face of a viral rebound. Latent HIV-1 reservoir sites are critical to HIV-1 cure and eradication; and this is because these secluded sites of the body of PLHA are repertoire from which the general circulation is usually replenished (as aforesaid) with infectious HIV-1 especially when infected individuals are taken off ART $[2,10,13,16]$. Some of these reservoir sites which can either be cellular or anatomical in nature are summarized in (Table 1) [17-54]. They are majorly responsible for rebound viraemia commonly experienced by PLHA when taken off ART.

Cellular reservoir sites of HIV-1 includes a group

Table 1: Summary of some cellular and anatomical reservoir sites of latent HIV-1.

\begin{tabular}{|c|c|c|c|}
\hline $\begin{array}{l}\text { S/ } \\
\text { NO. }\end{array}$ & $\begin{array}{l}\text { Cellular } \\
\text { Reservoir }\end{array}$ & $\begin{array}{l}\text { Anatomical } \\
\text { Reservoir }\end{array}$ & References \\
\hline 1. & CD4+ T cells & Brain and ${ }^{*} \mathrm{CNS}$ & Okoye, Picker [4]; Petito [19]; Alexaki, et al. [18]; Falangola, Petito [20], \\
\hline 2. & ${ }^{*} T_{\text {FH }}$ cells & Sex gonads & Perreau, et al. [21]; Moukambi, et al. [22]; Politch, et al. [23]; Matusali, et al. [24], \\
\hline 3. & CD8+ T cells & Spleen & Eller, et al. [25]; Mercure, et al. [26]; Nolan, et al. [27]; Fletcher, et al. [28], \\
\hline 4. & ${ }^{*} \mathrm{~T}_{\mathrm{REG}}$ & Lymph nodes & $\begin{array}{l}\text { Estes, et al. [29]; Kleinman, et al. [30]; Tirumalasetti, Latha [31]; Bogoch, et al. [32]; } \\
\text { Cainelli, et al. [33], }\end{array}$ \\
\hline 5. & ${ }^{*} \mathrm{~T}_{\mathrm{SCM}}, \mathrm{T}_{\mathrm{CM}}, \mathrm{T}_{\mathrm{TM}}$ & Thymus & $\begin{array}{l}\text { Tabler, et al. [34]; Zhu, et al. [35]; Sallusto, et al. [36]; Courgnaud, et al. [37]; Kolte } \\
\text { [38], }\end{array}$ \\
\hline 6. & Macrophages & ${ }^{*} \mathrm{GALT} / \mathrm{GIT}$ & Koppensteiner, et al. [39]; Moir, et al. [40]; Nkuize, et al. [41]; Kotler [42], \\
\hline 7. & *FDCs & $\begin{array}{l}\text { Genital tracts and } \\
\text { fluids }\end{array}$ & Burton, et al. [43]; Heesters, et al. [44]; Coombs, et al. [45], \\
\hline 8. & NK cells & Lungs & Mikulak, et al. [46]; Leal, et al. [47]; Grommes, et al. [48]; Almodovar, et al. [49], \\
\hline 9. & Monocytes & Adipose tissue & $\begin{array}{l}\text { Avettand-Fènoël, et al. [50]; Lambotte, et al. [51]; Couturier, et al. [52]; Damouche, } \\
\text { et al. [53]; Hazan, et al. [54]. }\end{array}$ \\
\hline
\end{tabular}


of special compartments or cells of the body (in this case, HIV-1 infected individuals) to which HIV-1 has immense specificity and propensity to cleave to upon invasion of a suitable host, and thus maintain a long lasting population of latent HIV-1 or provirus even in the face of ART $[16,18]$. These sites have receptors that mediate HIV-1 attachment and final penetration of the host cell, which are both necessary prerequisite for the establishment of viral persistence, virulence and pathological conditions $[18,33]$. Cellular reservoir sites continue to serve as sources of new infection especially when HIV-1 infected individuals are taken off therapy. Typical examples of cellular HIV-1 cellular reservoirs include CD4+ $\mathrm{T}$ cells, macrophages, astrocytes, monocytes, $T_{F H}$ cells, and cells of the gonads $[16,18,55]$. However, the best characterized cellular HIV-1 reservoir is a small population of resting memory and naïve CD4+ T cells [54-56].

Anatomical HIV reservoirs refer to some selected organs or tissues of the body of PLHA where latent HIV1 or provirus is lurking away from potent antiretroviral and immunological attack $[45,48,57]$. They are actually the source of infectious virus which initiates new infection in PLHA especially during cessation of ART. The hiding place of latent HIV-1 in the infected host, how they manage to survive in such sanctuaries and how to manipulate therapeutic interventional strategies towards their cure and possible eradication from the host are important factors to consider in finding a functional cure for HIV-1 infection [55]. The lungs, spleen, liver, gastrointestinal tract, bone marrow, lymph nodes, brain, thymus, kidney and adipose tissue are some perceived anatomical sites where latent HIV-1 are lurking in the body of PLHA $[5,55]$.

Viral rebound is an occasional clinical episode in HIV1-infected patients, and this condition usually occurs when HIV-1 at their latent stage resurfaces in vivo (from their reservoir sites) even in the face of potent ART, but especially during the stoppage of ART $[7,17,58,59]$. Most patients infected with HIV-1 may a times stay off ART - owing to the notable side effects and some complicated dosing plan associated with the use of ART/HAART in the management and/or treatment for HIV-1. This scenario can give room for the rebound of viraemia in infected individuals once ART/HAART cessation is anticipated and executed. Viral rebound is a repeated viral load that is $>50$ copies $/ \mathrm{ml}$ of peripheral blood $[7,58,59]$. HIV-1 rebound leads to increase in the plasma viral load of HIV-1 infected individuals; and this sets in motion the clinical episodes that characterize new infections in PLHA. In Africa where health care facilities and research is still at a comatose state, the challenge of viral rebound in PLHA may not be properly handled and managed. And this can in part contribute to the severity of the disease condition in PLHA in the continent. Some of the major causes of viral rebound as previously reported include drug resistance in the
HIV strain, patient's poor compliance to therapy and/ or poor adherence to an HIV-1 treatment regimen and interruption of ART/HAART $[5,17]$.

Total cure for HIV-1 infection in humans may soon become possible if the current medical technologies and HIV-1 therapeutic strategies can be re-invented to effectively target and dislodge latent HIV-1 from their fortresses in the body especially from reservoir sites harbouring latently infected CD4+ T cells $[17,57,58,60]$. This could be achieved (in part) by harmonizing all current animal models and other cure strategies with putative findings with a view to understanding properly, the molecular, pathological and immunological mechanisms underlying the effective control of the disease in some select few individuals (known as elite controllers), while those with chronic HIV-1 infection continues to take medications to keep the viral load under control. Some of the ongoing novel approaches and strategies geared towards finding a functional cure for HIV-1 infection are discussed later on. The HIV-1 pandemic still remains very active with several new cases of the diseases emerging across the world especially in developing countries - where health systems is still in a comatose and helpless situation. Nonetheless, morbidities due to HIV-1 related infections continue to abound across the globe especially in the African continent where the disease burden of the pandemic is high $[61,62]$.

Poor research facilities and the dearth of technical know-how on HIV-1/HIV-2 research in developing countries (Africa in particular) have also contributed to the spread of the epidemic in the region since there are no meaningful and coordinated efforts by the authorities to track and report new cases of the diseases and even the spread of the disease across the continent. This continues to happen irrespective of the vast knowledge of HIV-1 transmission routes and infection. It is therefore of utmost importance that researchers on HIV-1/HIV-2 in Africa and leaders of the region pull together their resources and knowledge on the subject with a view to understanding the immunological and pathological disposition of the disease in the region, especially as it regards to HIV-2 - which is endemic in sub-Saharan Africa. This can be achieved more through collaborations with notable HIV-1 researchers and institutions in the developed economies; and partly by setting up more PRIs and high-throughput research facilities in the region. These facilities will also help African scientists to effectively study HIV-1, HIV-2 and other intractable diseases notable in the region such as Ebola, Malaria and Lassa Fever to mention a few. Taken together, the region will not only actively contribute to the global search for a cure for HIV-1, but will also be on the top in finding a permanent and/or sustained therapeutic solution to other intractable diseases in Africa. 
Ongoing Approaches for Functional HIV-1 Cure: Current ART is Limited in Action but Novel-Non-ART May Provide Answers to HIV1 Cure

Antiretroviral drugs (ARDs) are anti-HIV-1 drugs which are used clinically for the treatment of HIV-1 infections in humans. ARDs are often used in combinations in ART when HIV-1 infection is considered; and these agents' helps to suppress in vivo HIV-1 replication, thereby delaying the progression of HIV-1 infection to AIDS. Nonetheless, ARDs used in ART for HIV-1 infected individuals can only treat the infection but still lacks the capacity to cure the infection. The concept of curing and eradication therefore, is the major target and ambition of all HIV-1 related research - since these ongoing approaches are mainly focused on finding a functional cure for HIV-1 infection so that the virus can be possibly eradicated from the body of PLHA $[5,6,55,57]$.

ART has really gone a long way in providing succour and relief to the millions of people living with HIV1 around the globe in terms of easing the infectious process of HIV-1 in vivo; as well as preventing the possible risk of getting an opportunistic infection due to weakened immune system resulting from HIV-1 infection. But there is however, no functional cure yet for HIV/AIDS or its related simian immunodeficiency virus (SIV) counterpart that infects monkeys and other NHPs. This necessitates the urgent need to step up HIV/AIDS cure research strategies in order to help the millions of people living with the disease across the world; and who suffer from several immunological and neurological untoward effects resulting from current ART. Unfortunately, the search for a cure for HIV-1 and its other counterpart, HIV-2, which is notable in Africa is at its lowest ebb in the African continent, if not lacking. And this is largely attributable to the paucity of funding and unavailability of high-tech research facilities for such capital-intensive research. Since latency is the major impediment to finding a total cure for HIV infection $[7,8,17,63,64]$; it is therefore important to continue to study HIV-1 latency and their reservoir sites in PLHA so as to better understand the immunological, physiological and molecular mechanisms that help the virus dodge ART and host immunological attack. Such pursuit should also be steadily followed with intensive research on HIV-2 which is notable in Africa; and African scientists and the medical and academic institutions should be on the pioneering forum of such medical interventions. Some of the major cure strategies for HIV-1 infection which include shock and kill strategies, silencing and gene therapy approaches are highlighted in this section.

\section{The shock strategy}

This strategy has been postulated or hypothesized in some quarters as a possible hope and futuristic cure approach for HIV-1 infection [65]. The major aim of the shock strategy is to upset or agitate cellular and anatomical reservoir sites of latent HIV-1 so that the hiding virus can be extricated from its hiding place in the infected host $[15,58]$. And this is putative and important for possible HIV-1 cure since current ART only limit the replication of the virus in vivo, but lacks the capacity to eradicate the virus (particularly from their hiding places in some cellular and anatomical sites) $[17,55]$. The HIV provirus or viral genome still remains integrated into the genome (DNA) of the infected host cell (particularly memory CD4+ T cells); and thus the host's immune system or ART cannot reach the provirus in these reservoir sites [55]. And the virus continues to maintain some level of viral replication (even in the face of ART) which is responsible for the persistent chronic infection in PLHA. In a recent study, it was reported that vorinostat, a histone deacetylase inhibitor (HDACi) has the capacity to activate HIV genes in vivo in HIV1 infected individuals on ART, thereby leading to the production of viral mRNA - which is important for HIV1 treatment [65]. The activation of the provirus in its reservoir sites cum the production of viral RNA will help to spark up immunological response in the host to flush out the hiding virus. HDACis are currently being investigated in the shock strategy as a potent tool for the breaking up of the latent HIV-1 reservoir in vivo - so that the quiescent virus can be targeted by both ART and host immune system for possible eradication [65]. Vorinostat (also known as suberoylanilide hydroxamic acid) has the capacity to activate quiescent HIV-1 in reservoir sites $[65,66]$. Initial infection with HIV-1 depletes the CD4+ helper T cells (which is central to the activation of other immune system cells), and this leaves the immune system incapacitated to handle other infections that result from HIV-1 invasion of the host cell [4]. This predisposes the infected individual to opportunistic infections; and a protracted inflammation and incapacitation of other immune system cells finally leads to the AIDS stage of the disease. But with the shock strategy, the reservoir sites of latent HIV-1 can be stimulated using LRAs such as growth factors (IL15); checkpoint blockades (PD-1); HDACis, and protein kinase $C(P K C)$ in order to release the latent virus so that they will be readily available for immune system attack $[3,6,7,67-69]$. The mechanisms of action of these agents are beyond the scope of this review.

\section{The kill strategy}

After reactivating the quiescent virus from their reservoir sites in vivo, what next? In as much as the shock strategy helps to reactivate the latent virus in their reservoir sites, by causing the virus to resume viral replication (which is an important factor for immunological attack), it is also important that these reservoir sites are efficiently purged of the infectious virus and targeted by host immune system for possible killing. Prior to HIV-1 infection, individuals possess 
highly-specific CD8+ T cells (known as cytotoxic T cells) which can target and kill intracellular parasites including HIV-1; but following infection with HIV-1, the population of the CD8+ T cells becomes depleted and loses their immunological effectiveness overtime $[59,60,70]$. Thus, the host becomes incapacitated to handle and control HIV-1 infection using its own immunological armamentarium. In the kill strategy, it is anticipated that the immune system of the hosts is boosted to effectively attack, kill and destroy the dislodged latent HIV-1 emanating from the reservoir sites [3,67-69]. In the course of persistent and/or chronic HIV-1 infection, the hosts immune system loses the battle to effectively fight the virus and other co-morbidities associated with the disease; and this is due mainly to the fact that the major aspect of the immune system that should have done the fight (i.e., the CD4+ T cells) have already been arrested and taken over by the invading virus. Since no functional cure for HIV-1 infection will be successful without a boosting of the infected host's immune system to take care of co-morbidities of the disease as well as the dislodged latent virus; it becomes important to combine the cure strategies with approaches that will help restore the lost immunity of the PLHA. This is the main mechanism of the proposed kill strategy for HIV1 cure. The current cure strategies of HIV-1 research cum kill strategy of HIV-1 cure looks to reactivating and making the immune system more effective in its role of locating and destroying pathogens including latent HIV-1 $[3,67,68,69]$. During HIV-1 infection, the host's immune system produces certain type of antibodies known as broadly neutralizing antibodies (bNAbs) whose function it is to eliminate HIV and possibly other viral pathogens [71]. But bNAbs, which are produced in high amount during HIV-1 infection, are not able to perform its statutory role of identifying and destroying HIV-1 after invasion because of the notable evolution and/or mutation rate of the virus $[69,70,72]$. The use of bNAbs is a typical example of fighting the virus using immunotherapy techniques. bNAbs, natural killer (NK) cells, toll-like receptor (TLR) agonists and the use of $T$ cells with chimeric antigen receptors (CARs) are some proposed approaches used in the kill strategy of HIV-1 cure $[67,71]$.

\section{The silence strategy}

The silencing strategy is geared towards using molecular therapeutic techniques that involves the inclusion of RNA interference (RNAi) to silence HIV-1, possibly shutting down its replication in vivo [72] RNAi is a biological process that occurs in both mammalian and non-mammalian cells; and it is a novel therapeutic strategy that is being proposed to be used against HIV1 infection because of its potential to stop translation (or gene expression) in a cell via the neutralization of targeted mRNAs $[72,73]$. RNAi is a naturally occurring mechanism for gene silencing induced by the presence of short interfering RNA (siRNA) molecules; and it is mainly used to knockdown the expression of RNA molecules [72,73]. It is an endogenous catalytic pathway that is triggered by double-stranded RNA (dsRNA); and the trigger can occur naturally, as in the case of a cellular infection by a dsRNA virus, or by the intentional introduction of dsRNA to induce user-directed degradation of the cognate transcript [72]. Since RNAi has the capacity to knockdown the expression of RNA molecules; it therefore might hold the potential to permanently stop latent-HIV-1 in reservoir cells from replicating, thereby preventing the production of infectious virus - which is responsible for the persistent infection seen occasionally in PLHA when taken off-ART. Certain agents such as cyclosporin $A$ are also being investigated for their potential to silence and stop the replication of HIV-1 in vivo. As aforementioned, latent HIV-1 is transcriptionally silent but replication competent; therefore, the virus has the potential to resume replication upon discontinuance of ART or any antiviral agents that temporarily stopped or reduced its in vivo replication. With RNAi, HIV-1 can possibly be silenced naturally, thus achieving the purpose of viral remission [72,73]. However, the therapeutic effect of this approach has not been clinically experimented or approved on PLHA; and this also applies to other proposed HIV-1 cure strategies highlighted in this review. It is therefore important to continue to study RNAi as a possible HIV-1 remission approach in order to get the full benefit of this natural way of curing or eradicating HIV-1 infection in PLHA. The targeting of the Vif protein of HIV-1 is one aspect of the HIV-1 cure strategy that is close to becoming a clinical reality in the treatment of HIV-1 infection [14]. In this silencing strategy, the Vif protein is targeted to activate the APOBEC antiviral pathway. APOBECs are deaminases that target the virus during reverse transcription (RT) to hypermutate the virus to silence any latent viruses activated by LRAs [14]. HIV-1 Vif protein inhibits APOBECs by preventing their viral packaging and shuttling them to degradation but therapies that target Vif protein of the virus would allow APOBECs to be packaged into newly activated latent virus and prevent further infection or reservoir formation [14]. The activation of the APOBEC antiviral pathway by targeting the Vif protein of HIV-1 is yet another putative strategy geared towards HIV-1 cure.

\section{Curing HIV-1 through gene therapy and transplan- tation}

Gene therapy allows pathological conditions to be treated at the molecular (gene) level through the administration of therapeutic genetic molecules (DNA or RNA) in order to improve and/or correct the genetic factors at play in a particular disease condition. Following the success story of HIV-1-free remission in the absence of ART for over a decayed in the Berlin patient (Timothy Ray Brown) after successful stem cell transplantation $[74,75]$, it was believed in some quarters that HIV-1 
remission or cure is possible, and should be attempted experimentally and clinically. Till date, Timothy Ray Brown (which will be known in the greater part of this review as the 'Berlin patient') still remains the only individual in the world, who is considered to have been cured of HIV-1 infection [74-76]. Cell and gene therapies offer the promise of preventing progressive HIV infection by interfering with HIV-1 replication in the absence of chronic antiviral therapy [76]. The Big Scientific question is: "Can the functionality of the Berlin patients' cure strategy be successfully recapitulated to help other HIV-1 infected patients around the globe"? While it may not be possible to recapitulate the Berlin patients' stem cell therapeutic approach in the millions of PLHA around the globe - owing to the clinical side effects (such as apparent tissue rejection), availability of suitable donors, and high cost of undertaking such clinical intervention; gene therapy and other futuristic novel experimental/clinical approach may yet provide a cure for HIV-1 if persistently pursued and approved. In the case of the Berlin patient who received an allogeneic hematopoietic stem cell (HSC) transplant from a homozygous CCR $5 \Delta 32$ donor, HIV-1 replication was perfectly inhibited and the patient has continued to stay off-ART without the detection of any active, replicating HIV-1 [76]. How this medical feat in the "fight against HIV-1" was made possible is very important for the medical community and the entire HIV community - since we all look forward hopefully, for a cure for HIV1. Achieving HIV-1 cure through transplantation (as was successfully exemplified in the Berlin patient) may not be feasible - owing to the aforementioned drawbacks associated with grafting techniques. Since HIV-1 enters the host cell through the combined efforts of a CD4 receptor and a chemokine co-receptor, particularly CCR5, gene therapy techniques may further provide suitable experimental/clinical approaches that could be exploited to provide some therapeutic protective functions to the host especially antiviral immunity against R5-tropic HIV-1 strains [75]. The use of gene therapy techniques as a potential cure strategy for HIV infection has been previously reviewed [75,77].

In summary, irrespective of the potentials of these cure strategies currently being investigated for curing and eradicating HIV-1 from the body of PLHA, it is also very important to consider the therapeutic efficacy of these agents in line with their untoward effects on the patients so that a better and much appreciable prognosis will be derived from their usage overtime. Nonetheless, Africa as a continent with a huge burden of HIV-1/2 incidence is still lagging behind in putting up novel research and development geared towards finding a cure for HIV/AIDS. And this can be due in part to the paucity of funding for such research, lack of infrastructure and lack of state of the art research facilities as well as HIV-1/2 researchers in the continent.
Biological Model Systems Used for HIV/AIDS

\section{Research}

This section is not exhaustive of the myriad of animal (biological) model systems that are used globally for the study of HIV/AIDS pathogenesis, immunology, transmission, prevention and treatment. Animals including NHPs are important in the HIV/AIDS research because they are predictive models of human hosts for research into diseases that affect human population [78]. Animal experimental models are mostly used for HIV/AIDS related research including those targeted at possible HIV/AIDS cure because of the seeming physiological, morphological and anatomical similarities that these animals or biological systems share with humans. Notably, these (biological) models also provide avenues through which novel medications and vaccines targeted at HIV-1 infection in PLHA can be properly evaluated even before such studies can be scaled-up clinically to the human population in clinical practice [78]. It will also be very wrong ethically to test novel HIV-1 medications and vaccines or other therapeutics for infectious disease treatment directly on humans; and this is why biological (animal) models are very important to HIV/AIDS research. The use of animals for biomedical research is important, but the welfare of the animals should be considered and prioritized in line with the research [80]. Minimal number of animals should always be used, and they should be used only in cases where there is no other alternative to the experimentation [79]; and such a strategy will help to save the lives of these animals and prevent their extinction.

This is why the 3 Rs (replacement, reduction, and refinement) is always a guiding principle in the humane use of animals for biomedical/scientific research. The use of animals therefore should be replaced with alternative models or avoided entirely (replacement); the number of animals used for scientific research should be as minimal as possible (reduction); and the welfare of animals used in biomedical research should be improved on while ensuring that animals suffer minimal pain in the course of the experiment (refinement) [79]. According to Fenwick, et al. [79], the three Rs are the tenets that guide the use of animals for biomedical/scientific research. This tenet is grounded in the premise that animals should be used only if a scientist's best efforts to find a non-animal alternative have failed, and that when animals are needed, only the most humane methods should be used on the smallest number of animals required to obtain valid information [80] for the anticipated research.

The biological (animal) models used for HIV/AIDS related research includes but not limited to SIV models, humanized mouse models, feline models, NHP models, transgenic mouse models and cell and tissue culture models. HIV-1 does not cause disease in other animal 
species because it is a species-specific virus that only causes infections in humans [80]. Nevertheless, the study of HIV-1 infections cannot be started in humans, but rather in other related animal models that also bear similar physiological, immunological and anatomical resemblances with their human counterparts. And since HIV-1 only replicates or causes infections in humans, some other animals such as the humanized mice can be re-engineered especially through transplantation techniques to start expressing some human cells or tissues that could be used to study the disease process of the virus. Though these models are not without some deficiencies since they are not $100 \%$ human-related hosts (in which HIV-1 causes infection), they also provide a suitable means through which HIV/AIDS and all of its associated clinical manifestations could be properly studied prior to the scaling-up and implementation of such research findings to the human host in clinical practice. Most importantly, these animal (biological) models allow HIV/AIDS scientists/researchers to do in vivo experimentations that would not have been possible in the first place using human host. The unique qualities of some of these animal models are succinctly reviewed in this section.

\section{Non-human primate model}

NHP models have immense research values for the testing of potential HIV-1 cure agents and vaccines. They also provide the means through which HIV1 immunopathogenesis can better be understood especially as it relates to the finding of a cure for the dreaded disease (AIDS) caused by the virus. Therefore, NHP represent an important tool and animal model for the HIV/AIDS cure research community. NHP models including monkeys, African green monkeys, macaques provides a suitable means of investigating the pathogenesis, immunology and immunohistochemistry of the HIV disease; and this is usually due in part to the genetic, physiological and anatomical relatedness of the NHPs and their human counterparts [81-83]. Several studies have shown that the infection of NHPs with the virulent strain of simian-human immunodeficiency viruses (SHIV) results in a similar disease that closely resembles HIV-1 infection in humans $[82,84]$. Rhesus macaque (RM) monkeys are usually the primary primate and/or NHP experimental animal models for HIV/SIV research [84]. However, HIV-1 (the causative agent of HIV infections in humans) replicates very poorly in these monkeys. Thus, HIV-1 pathogenesis cannot be studied directly using these monkeys. Instead, SIV, the NHP relative of HIV-1 is currently being used to study HIV1 pathogenesis in RMs due to the similarities of these two viruses (i.e., SIV and HIV); and many laboratories and ours now have HIV-1 models (for example, HIV1 macaque tropic, HIV-1mt strains) that can infect monkeys (RMs for example) and initiate similar clinical episodes associated with HIV-1 pathogenesis in humans [81]. These animal models are important tools for
HIV/AIDS research especially in the valuable data that they contribute towards finding a functional cure for the infection/disease. Most of the important features of HIV-1 pathogenesis and transmission have been understood through research carried out in NHPs [83], which are also infected with SIV, another relative of HIV. And NHPs are also important research tools for studying other infectious diseases of man. Nonetheless, the use of NHP for HIV1/2 research is still at its lowest ebb due to poor funding and unavailability of research facilities for such studies.

\section{Humanized mice (BLT mice)}

Humanized mice are mice species produced through the transplantation of CD34+ stem cells and/ or implantation of human tissue into immunodeficient mice $[80,85]$. Humanized mice carry functional human tissues, genes and cells. Such mice go on to generate the human B cells and myeloid cells; and they are also reengineered and implanted with human thymus tissues as well as bone marrow and liver cells $[80,85,86]$. And as a result, such mice go on to produce typical human cells and/or tissues which are vital for HIV/AIDS research. Humanized mice are also called BLT mice - because they produce human bone marrow, liver and thymus cells. BLT mice are capable of replicating the HIV-1 and HIV-2 strains in the human immune cells that they now harbor $[80,85]$; and this helps HIV/AIDS researchers to study the pathogenesis and other aspects of HIV. The functionality of BLT mice in supporting the replication of HIV-2, a notable viral pathogen of HIV in sub-Saharan Africa is promising, since humanized mice can be reengineered and utilized for studying the pathogenesis of HIV-2 in Africa. BLT mice can also be used in other biomedical research applications such as cancer research and research into other genetic diseases of humans; and they provide suitable avenue for the testing novel therapeutics as well $[80,85]$. Humanized mice carry different components of the human haematolymphoid system such as the liver, bone marrow and thymus cells $[8,10,85,86]$; and thus, they are still excellent animal models for specific investigations into HIV/AIDS related research.

\section{Feline models}

Felines refers to species of cat including domesticated cats. They are infected by a different type of retrovirus/lentivirus known as feline immunodeficiency virus (FIV) $[87,88]$. FIV is the only non-primate virus to cause AIDS-like disease in felines; and thus, can compromise the immune system of cats and cause immunodeficiency disease $[87,88]$ that is akin to AIDS in humans. Felines are often used in HIV/AIDS related research because of some similarities between HIV and FIV. But unlike HIV1; FIV infection in felines is naturally controlled in these non-primates [87,88]. More so, FIV pathogenesis is also similar to HIV infection in humans, thus warranting its use in HIV/AIDS research [87]. Feline models used for 
HIV/AIDS research include cats and other felines which are known to be naturally infected with FIV. However, felines have certain limitations; and are thus not widely used for HIV/AIDS related research even though some aspects of HIV-1 research can be attempted using the feline models $[87,88]$. Most importantly, feline models use the CD134 receptor instead of CD4 receptor as the major binding site for HIV-1 infection and entry into the cell [88]. This makes the target cells for FIV different from that of HIV-1 and its SIV counterpart. An understanding of the FIV model as a possible alternative model in the HIV/AIDS research may expose other striking features that may contribute positively towards finding a cure for HIV/AIDS.

In summary, researchers working on HIV-1/2 in Africa should as a matter of urgency localize the animal models that is best fit for HIV research in the region; and owing to the vast forest and wild life reserve in Africa, experiments in which animals are involved should be anticipated and carried out based on international protocol in order to secure the life and prevent the extinction of these animals.

\section{The Global HIV/AIDS Distribution: Africa in Fo- cus, and the Way Forward}

The need for more PRIs and high-throughput infectious disease laboratories in the African continent cannot be over emphasized. First, HIV-1 is a major concern in Africa particularly in the sub-Saharan part of Africa - where HIV-2 is most prevalent. Secondly, Africa is known for some peculiar infectious diseases such as Lassa fever, malaria and Ebola that ravages the people of the continent. More so, despite the current fight against ending HIV-1 infection and its scourge on the human race, new cases of the disease are still emerging and yet there is still no functional cure yet for the disease. HIV-1 has infected over 60 million people across the world since over 3 decades the infectious virus was first discovered; and over 30 million people have also died as a result of HIV-related complications [89]. According to the World Health Organization (WHO), the Sub-Saharan Africa remains most severely affected with HIV infection, with nearly 1 in every 25 adults (representing about $4.2 \%$ of her population) living with HIV and accounting for nearly two-thirds of the people living with HIV worldwide [89]. This development is not a palatable report - owing to the fact that it represents millions of people who may be in their prime stage of life. And with the poor state of health in most African nations, it is important for the African leaders to take the bull by the horn and rise up to the occasion of being at the fore front of fighting the infectious diseases headon. This can be done by coming up with legislation to back up the establishment and funding of facilities and researchers working on HIV-1/2 and other intractable diseases in the continent.

There are a lot of ways that the African nations can achieve this, but first of all, Africa is in dire need of high-tech research laboratories and research scientists who will collaborate and do world-class research with their counterparts across the world in order to find a lasting cure to this pandemic. With Africa having one of the most worsened cases of the disease, and with the poor healthcare facilities and research laboratories in many parts of the region, now is the time to act. According to the United Nations Programme on HIV and AIDS (UNAIDS), there were approximately 36.7 million people worldwide living with HIV/AIDS at the end of $2016[90,91]$. Of these, 2.1 million were children (< 15-years-old); and an estimated 1.8 million individuals worldwide became newly infected with HIV in 2016, thus accounting for about 5,000 new infections per day $[90,91]$. And this includes 160,000 children ( $<15$ years) who are also infected $[90,91]$.

Most of these children live in sub-Saharan Africa and were infected by their HIV-positive mothers during pregnancy, childbirth or breastfeeding $[90,91]$. Currently only $60 \%$ of people with HIV know their status [89-91]. The remaining 40\% (over 14 million people) still need to access HIV testing services [90,91]. As of July 2017, 20.9 million people living with HIV were accessing ART globally, up from 15.8 million in June 2015, 7.5 million in 2010, and less than one million in 2000 [90,91]. One million people died from AIDS-related illnesses in 2016, bringing the total number of people who have died from AIDS-related illnesses since the start of the epidemic to 35.0 million $[90,91]$. The vast majority of people living with HIV are in low- and middle-income countries. Sub-Saharan Africa is the most affected region, with an estimated 25.6 million people living with HIV in 2015; and about $66 \%$ of new HIV infections in 2015 occurred in sub-Saharan Africa [90,91].

According to Dube, et al. [92], about 42.9 million people are living with HIV across the globe; and approximately 2.1 million new infections of the disease occurred as of the year 2016. It is possible that newer cases might have emerged especially in poor resource countries and/or continents such as Africa where the disease is most prevalent. Though ARDs and/or ARTs have significantly reduced to a bearable minimum the percentages of HIV-1 co-morbidities, and thus ensured some level of comfort and improvement of the health status of those living with the disease, there is still no current cure or vaccine for the eradication of HIV-1 from the body of those living with the virus. On the other hand, the African continent is lacking in financial resources to fight its own HIV/AIDS issues especially by financing programs and interventions targeted at preventing the spread of the disease and proper management of already infected individuals. It is pitiable that most of the resources for fighting HIV/AIDS in Africa are mainly from donor agencies and foreign governments; and these measures cannot be sustainable owing to the changing economic situations around the globe. 
With Africa being the most hit region of the world with the HIV/AIDs epidemic, the situation varies across the countries in the continent. According to the United Nations Programme on HIV/AIDS (UNAIDS), the Eastern and Southern African countries happens to be the region hardest hit by HIV-1 - with about 19.4 million people living with HIV and 790,000 new HIV-1 infections; and South Africa accounted for about one third $(270,000)$ of the region's new infections $[90,91]$. In the Western and Central African states, the situation is pretty less severe when compared to the East and Southern African states. According to UNAIDS, HIV in West Africa and Central Africa is less prevalent than in East and Southern Africa $[90,91]$. There were about 6.1 million people living with HIV-1 in West and Central Africa as at 2016, and about 370,000 new infections occurred as at the time, with about half a million cases occurring in children $[90,91]$. HIV prevalence among adults in West and Central Africa is about $2.2 \%$, which is relatively low when compared to the East and Southern Africa where HIV prevalence in adults is about $7 \%[90,91]$. However, the prevalence of HIV in the Western and Central African states varies from one country to another. For example, the prevalence of HIV among adults in Nigeria is much less (2.9\%) than other African countries such as South Africa and Zambia where the prevalence is over $10 \%[90,91]$. In North Africa, the prevalence of HIV is relatively low (less than $1 \%$ ) compared to other regions of Africa $[90,91]$.

The factors spurring the prevalence and emergence of new HIV-1 infection in Africa is multifactorial. Many people living with HIV-1 in Africa are still unaware of their status. The fear of knowing their status has prevented many people from getting tested and seeking help especially through counseling and recruitment onto the ART/HAART treatment program. In addition, the low levels of access to ART treatment in most parts of Africa (especially in the rural areas) may also contribute to the morbidity and mortality associated with the disease. The stigma associated with HIV/AIDS may also contribute in preventing many from coming out to declare their status; and this means that many that is infected still remains largely unknown in the region because they fail to get tested or declare their status and get treatment. More so, most people infected with HIV-1 in sub-Saharan Africa, where the disease burden of HIV is highest may be without proper treatment care for the disease. Public awareness through sensitization of the public and creating more HIV/AIDS counseling and testing centers across the regions especially in the rural areas can help more people get tested and seek help where they need one. This will go a long way in helping to reduce the burden of the disease in the region especially by preventing the emergence of new infections and improving the lives of those already infected by the virus. With the sustainable developing goals (SDGs) hoping to end the AIDS epidemic by 2030, it is pertinent that Africa pulls her resources together to join in this quest since she bears the largest burden of the global HIV/AIDS epidemic.

\section{PRI: Minding the Research Gap in Africa}

$\mathrm{PRI}$ is a research and academic based institute cum laboratory facility that is mainly focused towards primatology, the scientific study of primates. In part, PRIs are also focused at understanding the physiological, metabolic, biological, and behavioural and socioecological roles and functions of primates (including NHPs and monkeys) in the environment and how data obtained from such studies can be extrapolated to humans. Since most research targeted at humans may be ethically wrong and impossible to undertake in humans, such as the testing of a new drug or treatment; primates are often the choicest of animal models to use for such experimentations owing to the genetic and evolutionary similarities that may exist among primates and humans. Nonetheless, there is paucity of PRIs and other high-tech infectious disease laboratories for studying Africa's own diseases especially HIV-1 (which she shares the greatest burden of the disease) as well as HIV-2 (common in the West African states). The Association of Assessment and Accreditation of Laboratory Animal Care (AAALAC) is the organization that promotes the humane treatment of animals (inclusive of primates and NHPs) in science through voluntary accreditation and assessment programs related to animal research and primatology in particular. In Africa, the Institute of Primate Research (IPR) in Nairobi, Kenya is the first organization in Africa to obtain the AAALAC International accreditation (www. aaalac.org) in late 2017. This adds to the two other PRI facilities in North-West University, South Africa and another US Naval facility that undertake such research in Mauritius. In all, Africa can only boast of three (3) PRI which is not bad but insufficient to encourage and engage her scientists to undertake world class research that will tackle head-on, the continents own diseases such as Ebola, Lassa fever and malaria that have held many Africans hostage.

\section{Concluding Remarks: Bridging the Research Gap of HIV-1 Cum HIV-2 in Africa}

This review is only but a brief on the subject of HIV-1 reservoir and cure research strategies and why Africa as an important beneficiary of the HIV/AIDS disease burden must bridge the lacuna of HIV/AIDS related research in the region. Basic research into HIV-1 including HIV-2 that is common in some African states is important to understanding the infection, pathogenesis, transmission and cure strategies of HIV-1 and HIV-2. Having state of the art research facilities and increased number of PRIs in the continent will help to spur and sustain a chain of research into HIV-1 and HIV-2 as well as other infectious diseases of Africa such as malaria, Lassa fever and Ebola to mention a few. More so, these facilities and funding will enable HIV-1/2 researchers in Africa to collaborate and sustain this research 
and others that are targeted at finding a sustainable solution to many intractable disease in Africa. For Africa to benefit sufficiently from the gains and spoils of war in terms of finding a cure or vaccine for HIV/AIDS, it must step up its support for HIV/AIDS related research in the continent. The prospects and potentials of having more PRIs and high-tech infectious disease laboratories in Africa especially in Nigeria (that primes itself as the Giant of Africa) cannot be overemphasized. Africa ranks highest in the HIV/AIDS pandemic. Most countries in the region are also noted for low standards of health and even low life expectancy due to both health and non-health related issues. And there is plethora of intractable infectious diseases that currently bedevil the wellbeing of the populace of Africa aside HIV/AIDS. This calls for urgent attention. Drawing from the quest to achieve the SDGs as other continents of the world, and with the One Health advocacy of WHO in the global community, it is important that Africa regains its place and pride of ensuring quality healthcare services for its huge populace by developing and building research centers and institutes that will help in addressing some of the disease's notable for the region. It is now time for Africa to wake up from its slumber and draw clues from countries in Asia, America and Europe who are doing outstanding research geared towards the treatment and possible control of numerous diseases including HIV/AIDS that mostly affects the African continent.

\section{Author's Contribution}

This article was conceived and designed by Chika Ejikeugwu and Hirofumi Akari with inputs, comments and guidance from Peter Eze, Ifeanyichukwu Iroha, Charles Esimone and Michael Adikwu.

\section{Acknowledgment}

This study was made possible by the generous funding made available by the Matsumae International Foundation (MIF), Tokyo, Japan. And we remain indebted to MIF and the laboratory of Professor Hirofumi Akari (D.V.M; Ph.D.) of Kyoto University, Kyoto, Japan for academic tutelage and research collaboration.

\section{Source of Funding}

Chika Ejikeugwu (Ph.D.) was funded by a postdoctoral fellowship grant (from MIF, Japan) to the Laboratory of Infectious Disease Model, Institute for Frontier Life and Medical Sciences, cum Center for Human Evolution Modeling Research, Primate Research Institute, Kyoto University, Kyoto, Japan.

\section{Competing Interest}

The authors declare that they have no competing interest.

\section{References}

1. Ciccaglione AR, Miceli M, Pisani G, Bruni R, ludicone $P$, et al. (2010) Improving HIV-2 detection by a combination of serological and nucleic acid amplification test assays. Journal of Clinical Microbiology 48: 2902-2908.

2. Soriano V, Gomes $P$, Heneine $W$, Holguin A, Doruana M, et al. (2000) Human immunodeficiency virus type 2 (HIV2) in Portugal: Clinical spectrum, circulating subtypes, virus isolation, and plasma viral load. J Med Virol 61: 111-116.

3. Jones RB, Walker BD (2016) HIV-specific CD8+ T cells and HIV eradication. J Clin Invest 126: 455-463.

4. Okoye AA, Picker LJ (2013) CD4+ T cell depletion in HIV infection: Mechanisms of immunologic failure. Immunol Rev 254: 54-64.

5. Barton K, Winckelmann A, Palmer S (2016) HIV-1 reservoirs during suppressive therapy. Trends Microbiol 24: 345-355.

6. Cary DC, Peterlin BM (2016) Targeting the latent reservoir to achieve functional HIV cure. F1000Res 5.

7. Chun TW, Davey RT Jr, Engel D, Lane HC, Fauci AS (1999) Re-emergence of HIV after stopping therapy. Nature 401: 874-875.

8. Hoxie JA, June $\mathrm{CH}$ (2012) Novel cell and gene therapies for HIV. Cold Spring Harb Perspect Med 2: 007179.

9. Hutter G, Ganepola S (2009) Eradication of HIV by transplantation of CCR5-deficient haematopoietic stem cells. Scientific World Journal 11: 1068-1076.

10. Bashiri K, Rezaei N, Nasi M, Cossarizza A (2018) The role of latency reversal agents in the cure of HIV: A review of current data. Immunol Lett 196: 135-139.

11. Spivak AM, Planelles V (2018) Novel latency reversing agents for HIV-1 cure. Annu Rev Med 69: 421-436.

12. Margolis DM, Archin NM (2017) Proviral latency, persistent human immunodeficiency virus infection, and the development of latency reversing agents. J Infect Dis 215: S111-S118.

13. Scarborough RJ, Gatignol A (2017) RNA interference therapies for an HIV-1 functional cure. Viruses 10: E8.

14. Bennett RP, Salter JD, Smith HC (2018) A new class of antiretroviral enabling innate immunity by protecting APOBEC3 from HIV Vif-dependent degradation. Trends Mol Med 24: 507-520.

15. Darcis G, Kula A, Bouchat S, Fujinaga K, Corazza F, et al. (2015) An In-depth comparison of latency-reversing agent combinations in various in vitro and ex vivo HIV-1 latency models identified Bryostatin-1+JQ1 and Ingenol-B+JQ1 to potently reactivate viral gene expression. PLOS Pathog 11: e1005063.

16. Saksena NK, Wang B, Zhou L, Soedjono M, Ho YS, et al. (2010) HIV reservoirs in vivo and new strategies for possible eradication of HIV from the reservoir sites. HIV AIDS (Auckl) 2: 103-122.

17. Miles B, Connick E (2016) TFH in HIV latency and as sources of replication competent virus. Trends Microbiol 24: $338-344$

18. Alexaki A, Liu Y, Wigdahi B (2008) Cellular reservoirs of HIV-1 and their role in viral persistence. Curr HIV Res 6: 388-400.

19. Petito CK (2004) Human immunodeficiency virus type 1 compartmentalization in the central nervous system. J Neurovirol 10: 21-24.

20. Falangola MF, Petito CK (1993) Choroid plexus infection in cerebral toxoplasmosis in AIDS patients. Neurology 43: 2035-2040. 
21. Perreau M, Savoye AL, De Crignis E, Corpataux JM, Cubas $\mathrm{R}$, et al. (2013) Follicular helper T cells serve as the major CD4 T cell compartment for HIV-1 infection, replication, and production. J Exp Med 210: 143-156.

22. Moukambi $F$, Rodrigues $V$, Fortier $\mathrm{Y}$, Rabezanahary $\mathrm{H}$, Borde C, et al. (2017) CD4 T Follicular Helper Cells and HIV Infection: Friends or Enemies? Front Immunol 8: 135.

23. Politch JA, Mayer KH, Anderson DJ (2016) HIV-1 is undetectable in preejaculatory secretions from HIV-1 infected men on suppressive HAART. AIDS 30: 1899-1903.

24. Matusali G, Dereuddre-Bosquet N, Le Tortorec A, Moreau M, Satie AP, et al. (2015) Detection of simian immunodeficiency virus in semen, urethra and male reproductive organs during efficient highly active antiretroviral therapy. J Virol 89: $5772-5787$.

25. Eller MA, Goonetilleke N, Tassaneetrithep B, Eller LA, Costanzo MC, et al. (2016) Expansion of inefficient HIVspecific CD8 T cells during acute infection. J Virol 90: 40054016.

26. Mercure L, Phaneuf D, Wainberg MA (1993) Detection of unintegrated human immunodeficiency virus type 1 DNA in persistently infected CD8+ cells. J Gen Virol 74: 2077-2083.

27. Nolan DJ, Rose R, Rodriguez PH, Salemi M, Singer EJ, et al (2018) The Spleen Is an HIV-1 sanctuary during combined antiretroviral therapy. AIDS Res Hum Retroviruses 34: 123 125.

28. Fletcher CV, Staskus K, Wietgrefe SW, Rothenberger M, Reilly C, et al. (2014) Persistent HIV-1 replication is associated with lower antiretroviral drug concentrations in lymphatic tissues. Proc Natl Acad Sci U S A 111: 23072312.

29. Estes JD, Li Q, Reynolds MR, Wietgrefe S, Duan L, et al. (2006) Premature induction of an immunosuppressive regulatory $T$ cell response during acute simian immunodeficiency virus infection. J Infect Dis 193: 703-712.

30. Kleinman AJ, Sivanandham R, Pandrea I, Chougnet CA, Apetrei C (2018) Regulatory T Cells as potential targets for HIV cure research. Front Immunol 9: 734.

31. Tirumalasetti N, Latha PP (2014) Lymph nodes cytology in HIV seropositive cases with haematological alterations. Indian J Med Res 139: 301-307.

32. Bogoch II, Andrews JR, Nagami EH, Rivera AM, Gandhi RT, et al. (2013) Clinical predictors for the aetiology of peripheral lymphadenopathy in HIV-infected adults. HIV Med 14: 182-186.

33. Cainelli F, Vallone A, Tanko MN, Vento S (2010) Lymph node and pathogenesis of infection with HIV-1. The Lancet Infectious Diseases 10: 71-72.

34. Tabler CO, Lucera MB, Haqqani AA, McDonald DJ, Migueles SA, et al. (2014) CD4+ Memory Stem Cells ( $T_{\mathrm{SCM}}$ ) are Infected by HIV-1 in a Manner Regulated in Part by SAMHD1 Expression. J Virol 88: 4976-4986.

35. Zhu J, Yamane H, Paul WE (2010) Differentiation of effector CD4 T cell populations. Annu Rev Immunol 28: 445-489.

36. Sallusto F, Geginat J, Lanzavecchia A (2004) Central memory and effector memory $T$ cell subsets: Function, generation, and maintenance. Annu Rev Immunol 22: 745-763.

37. Courgnaud V, Laure F, Brossard A, Bignozzi C, Goudeau A, et al. (1991) Frequent and early in utero HIV-1 infection. AIDS Res Hum Retroviruses 7: 337-341.

38. Kolte L (2013) Thymic function in HIV infection. Dan Med
J 60: 4622

39. Koppensteiner $\mathrm{H}$, Banning $\mathrm{C}$, Schneider $\mathrm{C}$, Hohenberg $\mathrm{H}$, Schindler M (2012) Macrophage internal HIV-1 is protected from neutralizing antibodies. J Virol 86: 2826-2836.

40. Moir S, Chun TW, Fauci AS (2011) Pathogenic mechanisms of HIV disease. Annu Rev Pathol 6: 223-248.

41. Nkuize M, De Wit S, Muls V, Arvanitakis M, Buset M (2010) Upper gastrointestinal endoscopic findings in the era of highly active antiretroviral therapy. HIV Medicine 11: 412417.

42. Kotler DP (2005) HIV infection and the gastrointestinal tract. AIDS 19: 107-117.

43. Burton GF, Keele BF, Estes JD, Thacker TC, Gartner S (2002) Follicular dendritic cell contributions to HIV pathogenesis. Semin Immunol 14: 275-284.

44. Heesters BA, Lindqvist M, Vagefi PA, Scully EP, Schildberg FA, et al. (2015) Follicular Dendritic Cells Retain Infectious HIV in Cycling Endosomes. PLoS Pathog 11: e1005285.

45. Coombs RW, Reichelderfer PS, Landay AY (2003) Recent observations on HIV type-1 infection in the genital tract of men and women. AIDS 17: 455-480.

46. Mikulak J, Oriolo F, Zaghi E, Di Vito C, Mavilio D (2017) Natural killer cells in HIV-1 infection and therapy. AIDS 31: 2317-2330.

47. Leal FE, Premeaux TA, Abdel-Mohsen M, Ndhlovu LC (1998) Role of Natural Killer Cells in HIV-Associated Malignancies. Front Immunol 8: 315.

48. Grommes J, Drechsler M, Soehnlein O (2014) CCR5 and FPR1 mediate neutrophil recruitment in endotoxin-induced lung injury. J Innate Immu 6: 111-116.

49. Almodovar S, Knight R, Allshouse AA, Roemer S, Lozupone $C$, et al. (2012) Human immunodeficiency virus nef signature sequences are associated with pulmonary hypertension. AIDS Research and Human Retroviruses 28: 607-618.

50. Avettand-Fenoel V, Hocqueloux L, Ghosn J, Cheret A, Frange $P$, et al. (2016) Total HIV-1 DNA, a Marker of Viral Reservoir Dynamics with Clinical Implications. Clin Microbiol Rev 29: 859-880.

51. Lambotte O, Taoufik Y, de Goer MG, Wallon C, Goujard C, et al. (2000) Detection of infectious HIV in circulating monocytes from patients on prolonged highly active antiretroviral therapy. J Acquir Immune Defic Syndr 23: 114-119.

52. Couturier J, Winchester LC, Suliburk JW, Wilkerson GK, Podany AT, et al. (2018) Adipocytes impair efficacy of antiretroviral therapy. Antiviral Res 154: 140-148.

53. Damouche A, Lazure T, Avettand-Fenoel V, Huot N, DejucqRainsford N, et al. (2015) Adipose tissue is a neglected viral reservoir and an inflammatory site during chronic HIV and SIV infection. PLOS Pathog 11: e1005153.

54. Hazan U, Romero IA, Cancello R, Valente S, Perrin V, et al. (2002) Human adipose cells express CD4, CXCR4, and CCR5 receptors: a new target cell type for the immunodeficiency virus-1? FASEB J 16: 1254-1266.

55. Churchill MJ, Deeks SG, Margolis DM, Siliciano RF, Swanstrom R (2016) HIV reservoirs: What, where and how to target them. Nat Rev Microbiol 14: 55-60.

56. Eugenin EA, Berman JW (2016) Improved methods to detect low levels of HIV using antibody-based technologies. Methods Mol Biol 1354: 265-279. 
57. Levy JA (2009) HIV pathogenesis: 25 years of progress and persistent challenges. AIDS 23: 147-160.

58. Watson DC, Moysi E, Valentin A, Bergamaschi C, Santhi Devasundaram, et al. (2018) Treatment with naïve heterodimeric IL-15 increases cytotoxic lymphocytes and reduces SHIV RNA in lymph nodes. PLOS pathogens 14 e1006902.

59. Jiao Yan-Mei, Liu Cui-e, Luo Li-Jing, Zhu Wei-Jun, Zhang $\mathrm{T}$, et al. (2015) CD4+CD25+CD127 regulatory cells play multiple roles in maintaining HIV-1 p24 production in patients on long-term treatment: HIV-1 p24-producing cells and suppression of anti-HIV immunity. International Journal of Infectious Diseases 37: 42-49.

60. Stein DS, Korvick JA, Vermund SH (1992) CD4+ lymphocyte cell enumeration for prediction of clinical course of human immunodeficiency virus disease: A review. J Infect Dis 165 : 352-363.

61. Iroha I, Nwakaeze E, Ejikeugwu C, Oji A, Udu-lbiam E, et al (2013) Frequency and antibiogram of uropathogens isolated from Urine Samples of HIV Infected Patients on Antiretroviral Therapy. American Journal of BioScience 1: 50-53.

62. Petrosilla N, Martini L, Raffaele B, Nicastri F, Bordi E, et al. (2002) Urinary tract infection in HIV-infected patients. International conference on AIDS 9: 7-14.

63. Lan P, Tonomura N, Shimizu A, Wang S, Yang YG (2006) Reconstitution of a functional human immune system in immunodeficient mice through combined human fetal thymus/ liver and CD34+ cell transplantation. Blood 108: 487-492.

64. Tarancon-Diez L, Dominguez-Molina B, Viciana P, LopezCortes L, Ruiz-Mateos E (2018) Long-term persistent elite HIV controllers: the right model of functional cure. EBioMedicine 28: 15-16.

65. Archin NM, Liberty AL, Kashuba AD, Choudhary SK, Kuruc JD, et al. (2012) Administration of vorinostat disrupts HIV1 latency in patients on antiretroviral therapy. Nature 487 : 482-485.

66. Margolis DM (2011) Histone deacetylase inhibitors and HIV latency. Curr Opin HIV AIDS 6: 25-29.

67. Halper-Stromberg A, Nussenzweig MC (2016) Towards HIV-1 remission: Potential roles for broadly neutralizing antibodies. J Clin Invest 126: 415-423.

68. Passaes CP, Sáez-Cirión A (2014) HIV cure research: advances and prospects. Virology 45: 340-352.

69. Battivelli E, Dahabieh MS, Abdel-Mohsen M, Svensson JP, Da Silva IT, et al. (2018) Distinct chromatin functional states correlate with HIV latency reactivation in infected primary CD4 T Cells. eLife 7: e34655.

70. Williams KC, Burdo TH (2009) HIV and SIV infection - the role of cellular restriction and immune responses in viral replication and pathogenesis. APMIS 117: 400-412.

71. Ananworanich J, McSteen B, Robb ML (2015) Broadly neutralizing antibodies and the HIV reservoir in acute infection: a strategy toward HIV remission? Curr Opin HIV AIDS 10: 198-206.

72. Farrell RE (2010) RNAi: Take a RISC - Role the Dicer. In: RNA methodologies, a laboratory guide for isolation and characterization. (4 ${ }^{\text {th }}$ edn), Academic Press, 539-560.
73. Carter M, Shieh J (2015) Identifying genes and proteins of interest. In: Guide to research techniques in neuroscience. ( $2^{\text {nd }}$ edn) , Academic Press 203-218.

74. Jessen H, Allen TM, Streeck H (2014) How a single patient influenced HIV research - 15 year follow-up. The New England Journal of Medicine 370: 682-683.

75. Allers K, Hutter G, Hofman J, Loddenkemper C, Rieger $\mathrm{K}$, et al. (2011) Evidence for the cure of HIV infection by CCR5D 32/D32 stem cell transplantation. Blood 117: 27912799.

76. Mueller MC, Bogner JR (2007) Treatment with CCR5 antagonists: which patient may have a benefit? Eur $\mathrm{J}$ Med Res 12: 441-452.

77. Chan E, Towers GJ, Qasim W (2014) Gene therapy strategies to exploit TRIM derived restriction factors against HIV-1. Viruses 6: 243-263.

78. Gardner MB, Luciw PA (1989) Animal models of AIDS. FASEB J 3: 2593-2606.

79. Fenwick N, Griffin G, Gauthier C (2009) The welfare of animals used in science: How the "three Rs" ethic guides improvements. Can Vet J 50: 523-530.

80. Garcia JV (2016) Humanized mice for HIV and AIDS research. Curr Opin Virol 19: 56-64.

81. Saito A, Akari H (2013) Macaque-tropic human immunodeficiency virus type 1: Breaking out of the host restriction factors. Front Microbiol 4: 1-13.

82. Nath BM, Schumann KE, Boyer JD (2000) The chimpanzee and other non-human-primate models in HIV-1 vaccine research. Trends Microbiol 8: 426-431.

83. Sui Y, Gordon S, Franchini G, Berzofsky JA (2013) Non human primate models for HIVIAIDS vaccine development. Curr Protoc Immunol 102: 1-30.

84. Hirsch VM, Olmsted RA, Murphey-Corb M, Purcell RH, Johnson PR (1989) An African primate lentivirus $\left(\mathrm{SIV}_{\mathrm{sm}}\right)$ closely related to HIV-2. Nature 339: 389-392.

85. McCune JM, Namikawa R, Shih CC, Rabin L, Kaneshima H (1990) Suppression of HIV infection in AZT-treated SCIDhu mice. Science 247: 564-566.

86. Akkina R (2014) New generation humanized mice for virus research: comparative aspects and future prospects. Virology 435: 14-28.

87. Burkhard MJ, Dean GA (2003) Transmission and immunopathogenesis of FIV in cats as a model for HIV. Curr HIV Res 1: 15-29.

88. Shimojima M, Miyazawa T, Ikeda Y, McMonagle EL, Haining $\mathrm{H}$, et al. (2004) Use of CD134 As a Primary Receptor by the Feline Immunodeficiency Virus. Science 303: 1192-1195.

89. World Health Organization, (WHO) (2017) Global Health Observatory (GHO) Data.

90. United Nations Programme on HIV and AIDS, (UNAID) (2017) The global HIV/AIDS epidemic.

91. https://www.hiv.gov/hiv-basics/overview/data-and-trends/ global-statistics

92. Dube K, Luter S, Lesnar B, Newton L, Jerome Galea, et al. (2018) Use of 'eradication' in HIV cure-related research: A public health debate. BMC Public Health 18: 245.

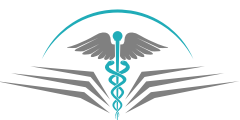

